IJMMS 29:1 (2002) 17-30

PII. S0161171202010955

http://ijmms.hindawi.com

(c) Hindawi Publishing Corp.

\title{
FIXED POINT AND COINCIDENCE POINT THEOREMS FOR A PAIR OF SINGLE-VALUED AND MULTI-VALUED MAPS ON A METRIC SPACE
}

\section{S. VENKATA RATNAM NAIDU}

Received 26 September 2000 and in revised form 5 March 2001

We obtain fixed point and coincidence point theorems for a pair of single-valued and multivalued maps on a metric space satisfying a generalized nonexpansive type condition.

2000 Mathematics Subject Classification: 47H10, 54H25.

Bogin [1] proved a fixed point theorem for a nonexpansive type self-map on a complete metric space. While Rhoades obtained a generalization of it (see [8, Theorem 1]) by replacing the constant coefficients in the governing inequality of the map with nonnegative real-valued functions of the independent variables, Ćirić [3] obtained a generalization by further weakening the governing inequality without allowing the coefficients to vary. Chandra et al. obtained a coincidence point theorem (see [2, Theorem 2.1]) for a pair of self-maps on a metric space unifying the results of Rhoades and Ćirić. They also obtained a corresponding version for multimaps (see [2, Theorem 2.2]). In this paper, we obtain proper generalizations of Theorems 2.1 and 2.2 of Chandra et al. [2].

Throughout, unless otherwise stated, $(X, d)$ is a metric space, $K(X)$ is the collection of all nonempty compact subsets of $X, \mathrm{CL}(X)$ is the collection of all nonempty closed subsets of $X, H$ is the extended Hausdorff metric on $\mathrm{CL}(X), F$ is a mapping from $X$ into $\mathrm{CL}(X), f, S$ are self-maps on $X, I$ is the identity map on $X$, for any self-map $h$ on $X, \mathfrak{R}(h)=\{h x: x \in X\}, \mathbb{R}^{+}$is the set of all nonnegative real numbers, $\mathbb{N}$ is the set of all positive integers, $\Omega:\left(\mathbb{R}^{+}\right)^{5} \rightarrow \mathbb{R}^{+}$is monotonically increasing in each coordinate variable, for any $t_{1}, t_{2}, t_{3}, t_{4}, t_{5} \in \mathbb{R}^{+}, \Omega\left(t_{1}^{+}, t_{2}^{+}, t_{3}^{+}, t_{4}^{+}, t_{5}^{+}\right)$ $=\inf \left\{\Omega\left(s_{1}, s_{2}, s_{3}, s_{4}, s_{5}\right): s_{j} \in\left(t_{j},+\infty\right)\right.$ for all $\left.j=1,2,3,4,5\right\}, \Omega\left(t_{1}, t_{2}^{+}, t_{3}^{+}, t_{4}^{+}, t_{5}^{+}\right)=$ $\inf \left\{\Omega\left(t_{1}, s_{2}, s_{3}, s_{4}, s_{5}\right): s_{j} \in\left(t_{j},+\infty\right)\right.$ for all $\left.j=2,3,4,5\right\}, \sigma_{j}: \mathbb{R}^{+} \rightarrow \mathbb{R}^{+}(j=1,2)$ and $\zeta: \mathbb{R}^{+} \rightarrow \mathbb{R}^{+}$are defined as

$$
\begin{gathered}
\sigma_{1}(t)=\Omega\left(0^{+}, 0^{+}, t^{+}, t^{+}, t^{+}\right), \quad \sigma_{2}(t)=\Omega\left(t, 0^{+}, 0^{+}, t^{+}, 0^{+}\right), \\
\zeta(t)=\max \left\{\sigma_{1}(t), \sigma_{2}(t)\right\},
\end{gathered}
$$

for all $t \in \mathbb{R}^{+}, \alpha, D$ are functions from $X \times X$ to $\mathbb{R}^{+}$defined as

$$
\begin{aligned}
& \alpha(x, y)=\Omega(d(S x, f x), d(S y, f y), d(S x, S y), d(f x, S y), d(S x, f y)), \\
& D(x, y)=\Omega(d(S x, F x), d(S y, F y), d(S x, S y), d(S y, F x), d(S x, F y)),
\end{aligned}
$$

for all $x, y$ in $X$. 
DEFINITION 1. We say that $(f, S)$ has property $A$ if there is a sequence $\left\{x_{n}\right\}_{n=0}^{\infty}$ in $X$ such that $S x_{n+1}=f x_{n}\left(=y_{n}\right.$, say) for all $n=0,1,2 \ldots$

LEMmA 2. Suppose that $(f, S)$ has property $A,\left\{d\left(y_{n}, y_{n+1}\right)\right\}_{n=0}^{\infty}$ converges to zero, $\sigma_{1}(t)<t$ for all $t \in(0, \infty)$, and that

$$
d(f x, f y) \leq \alpha(x, y)
$$

for all $x, y$ in $X$. Then $\left\{y_{n}\right\}$ is Cauchy.

Proof. If possible, suppose that $\left\{y_{n}\right\}$ is not Cauchy. Then there exists a positive real number $\varepsilon$ with the following property: given $N \in \mathbb{N}$ there exists $m, n \in \mathbb{N} \ni m>$ $n \geq N$ and $d\left(y_{n}, y_{m}\right) \geq \varepsilon$. Hence there exist strictly increasing sequences $\left\{n_{k}\right\}_{k=1}^{\infty}$ and $\left\{m_{k}\right\}_{k=1}^{\infty}$ in $\mathbb{N}$ such that $k<n_{k}<m_{k}, d\left(y_{n_{k}}, y_{m_{k}}\right) \geq \varepsilon$, and $d\left(y_{n_{k}}, y_{m_{k}-1}\right)<\varepsilon$ for all $k \in$ $\mathbb{N}$. Since $\left\{d\left(y_{n}, y_{n+1}\right)\right\}$ converges to zero, it follows that $\left\{d\left(y_{n_{k}}, y_{m_{k}}\right)\right\}_{k=1}^{\infty}$ converges to $\varepsilon$ and that for any fixed $r, s$ in $\{-1,0,1\}$, the sequence $\left\{d\left(y_{n_{k}+r}, y_{m_{k}+s}\right)\right\}_{k=1}^{\infty}$ also converges to $\varepsilon$. We have $\alpha\left(x_{n_{k}}, x_{m_{k}+1}\right)=\Omega\left(d\left(y_{n_{k}-1}, y_{n_{k}}\right), d\left(y_{m_{k}}, y_{m_{k}+1}\right), d\left(y_{n_{k}-1}, y_{m_{k}}\right)\right.$, $\left.d\left(y_{n_{k}}, y_{m_{k}}\right), d\left(y_{n_{k}-1}, y_{m_{k}+1}\right)\right)$ for all $k \in \mathbb{N}$. We note that the limit superior of $\left\{\alpha\left(x_{n_{k}}, x_{m_{k}+1}\right)\right\}_{k=1}^{\infty}$ is less than or equal to $\Omega\left(0^{+}, 0^{+}, \varepsilon^{+}, \varepsilon^{+}, \varepsilon^{+}\right)\left(=\sigma_{1}(\varepsilon)\right)$. We also note that $\left\{d\left(f x_{n_{k}}, f x_{m_{k}+1}\right)\right\}_{k=1}^{\infty}$ converges to $\varepsilon$. From (3) we have

$$
d\left(f x_{n_{k}}, f x_{m_{k}+1}\right) \leq \alpha\left(x_{n_{k}}, x_{m_{k}+1}\right)
$$

for all $k \in \mathbb{N}$. By taking limit superiors on both sides of (4) as $k \rightarrow+\infty$ we obtain $\varepsilon \leq \sigma_{1}(\varepsilon)$. This is a contradiction since $\sigma_{1}(t)<t$ for all $t \in(0, \infty)$ and $\varepsilon>0$. Hence $\left\{y_{n}\right\}$ is Cauchy.

Definition 3. We say that $\Omega$ has property $A$ if $\Omega(t, s, t, 0, t+s)<s$ for all $s, t \in \mathbb{R}^{+}$ with $t<s$.

DEFINITION 4. We say that $\Omega$ has property $B$ if there exist (i) a monotonically increasing function $\varphi: \mathbb{R}^{+} \rightarrow \mathbb{R}^{+}$with $\varphi\left(t^{+}\right)<t$ for all $t \in(0, \infty)$, and (ii) for each $t \in \mathbb{R}^{+}$a nonempty index set $I_{t}$ and nonnegative real numbers $\beta_{i}, \gamma_{i}\left(i \in I_{t}\right)$ such that $\sup \left\{\gamma_{i}: i \in I_{t}\right\}<1, \Omega(t, t, 2 t, t, t+s) \leq \sup \left\{\left(1+\beta_{i}\right) t+\gamma_{i} s: i \in I_{t}\right\}$ for all $s \in[t, 2 t]$, $\Omega\left(t, t, t, 0, \lambda_{t} t\right) \leq \varphi(t)$, where $\lambda_{t}=\sup \left\{\left(1+\beta_{i}\right) /\left(1-\gamma_{i}\right): i \in I_{t}\right\}$.

LEMмA 5. Suppose that $(f, S)$ has property $A, \Omega$ has properties $A$ and $B$, and that inequality (3) is true for all $x, y$ in $X$. Then $\left\{d\left(y_{n}, y_{n+1}\right)\right\}_{n=0}^{\infty}$ converges to zero.

Proof. We have $\alpha\left(x_{n}, x_{n+1}\right)=\Omega\left(d\left(y_{n-1}, y_{n}\right), d\left(y_{n}, y_{n+1}\right), d\left(y_{n-1}, y_{n}\right), 0, d\left(y_{n-1}\right.\right.$, $\left.y_{n+1}\right)$ ) for all $n \in \mathbb{N}$. From inequality (3) we have

$$
d\left(y_{n}, y_{n+1}\right) \leq \alpha\left(x_{n}, x_{n+1}\right)
$$

for all $n \in \mathbb{N}$. If $d\left(y_{m-1}, y_{m}\right)<d\left(y_{m}, y_{m+1}\right)$ for some $m \in \mathbb{N}$, then since $d\left(y_{n-1}, y_{n+1}\right)$ $\leq d\left(y_{n-1}, y_{n}\right)+d\left(y_{n}, y_{n+1}\right), \Omega$ is increasing in each coordinate variable and $\Omega$ has property $A$, it follows from (5) that $d\left(y_{m}, y_{m+1}\right)<d\left(y_{m}, y_{m+1}\right)$ which is a contradiction. Hence $d\left(y_{n}, y_{n+1}\right) \leq d\left(y_{n-1}, y_{n}\right)$ for all $n \in \mathbb{N}$.

From (3) we have

$$
d\left(y_{n}, y_{n+2}\right) \leq \alpha\left(x_{n}, x_{n+2}\right)
$$


for all $n \in \mathbb{N}$. But

$$
\begin{aligned}
\alpha\left(x_{n}, x_{n+2}\right)= & \Omega\left(d\left(y_{n-1}, y_{n}\right), d\left(y_{n+1}, y_{n+2}\right), d\left(y_{n-1}, y_{n+1}\right),\right. \\
& \left.d\left(y_{n}, y_{n+1}\right), d\left(y_{n-1}, y_{n+2}\right)\right) \\
\leq & \Omega\left(d\left(y_{n-1}, y_{n}\right), d\left(y_{n+1}, y_{n+2}\right), d\left(y_{n-1}, y_{n}\right)+d\left(y_{n}, y_{n+1}\right),\right. \\
& \left.d\left(y_{n}, y_{n+1}\right), d\left(y_{n-1}, y_{n}\right)+d\left(y_{n}, y_{n+2}\right)\right) \\
\leq & \Omega\left(d\left(y_{n-1}, y_{n}\right), d\left(y_{n-1}, y_{n}\right), 2 d\left(y_{n-1}, y_{n}\right), d\left(y_{n-1}, y_{n}\right),\right. \\
& \left.d\left(y_{n-1}, y_{n}\right)+d\left(y_{n}, y_{n+2}\right)\right)
\end{aligned}
$$

since the sequence $\left\{d\left(y_{k-1}, y_{k}\right)\right\}$ is monotonically decreasing and $\Omega$ is increasing in each coordinate variable. Since $\Omega$ has property $B$, there exist (i) a monotonically increasing function $\varphi: \mathbb{R}^{+} \rightarrow \mathbb{R}^{+}$with $\varphi\left(t^{+}\right)<t$ for all $t \in(0, \infty)$ and (ii) for each $t \in \mathbb{R}^{+}$a nonempty index set $I_{t}$ and nonnegative real numbers $\beta_{i}, \gamma_{i}\left(i \in I_{t}\right)$ such that $\sup \left\{\gamma_{i}: i \in I_{t}\right\}<1, \Omega(t, t, 2 t, t, t+s) \leq \sup \left\{\left(1+\beta_{i}\right) t+\gamma_{i} s: i \in I_{t}\right\}$ for all $s \in[t, 2 t]$, and $\Omega\left(t, t, t, 0, \lambda_{t} t\right) \leq \varphi(t)$, where $\lambda_{t}=\sup \left\{\left(1+\beta_{i}\right) /\left(1-\gamma_{i}\right): i \in I_{t}\right\}$. Since $d\left(y_{n}, y_{n+2}\right) \leq$ $d\left(y_{n}, y_{n+1}\right)+d\left(y_{n+1}, y_{n+2}\right) \leq 2 d\left(y_{n-1}, y_{n}\right)$, we have

$$
\begin{aligned}
& \Omega\left(d\left(y_{n-1}, y_{n}\right), d\left(y_{n-1}, y_{n}\right), 2 d\left(y_{n-1}, y_{n}\right), d\left(y_{n-1}, y_{n}\right), d\left(y_{n-1}, y_{n}\right)+d\left(y_{n}, y_{n+2}\right)\right) \\
& \quad \leq \sup \left\{\left(1+\beta_{i}\right) d\left(y_{n-1}, y_{n}\right)+\gamma_{i} d\left(y_{n}, y_{n+2}\right): i \in I_{r}\right\}
\end{aligned}
$$

provided $d\left(y_{n-1}, y_{n}\right) \leq d\left(y_{n}, y_{n+2}\right)$, where $r=d\left(y_{n-1}, y_{n}\right)$. We assume that $d\left(y_{n-1}\right.$, $\left.y_{n}\right) \leq d\left(y_{n}, y_{n+2}\right)$. Then, from (6), (7), and (8), we have

$$
d\left(y_{n}, y_{n+2}\right) \leq \sup \left\{\left(1+\beta_{i}\right) d\left(y_{n-1}, y_{n}\right)+\gamma_{i} d\left(y_{n}, y_{n+2}\right): i \in I_{r}\right\}
$$

Hence given $\varepsilon>0$ there exists $j \in I_{r}$ such that

$$
d\left(y_{n}, y_{n+2}\right) \leq\left(1+\beta_{j}\right) d\left(y_{n-1}, y_{n}\right)+\gamma_{j} d\left(y_{n}, y_{n+2}\right)+\varepsilon
$$

Hence in view of the hypothesis that $1>\sup \left\{\gamma_{i}: i \in I_{r}\right\}(=\mu$, say), we have

$$
d\left(y_{n}, y_{n+2}\right) \leq\left(\frac{1+\beta_{j}}{1-\gamma_{j}}\right) d\left(y_{n-1}, y_{n}\right)+\left(\frac{\varepsilon}{1-\gamma_{j}}\right) \leq \lambda_{r} d\left(y_{n-1}, y_{n}\right)+\left(\frac{\varepsilon}{1-\mu}\right) .
$$

Since $\varepsilon>0$ is arbitrary, from (11) it follows that

$$
d\left(y_{n}, y_{n+2}\right) \leq \lambda_{r} d\left(y_{n-1}, y_{n}\right)
$$

Since $\lambda_{r} \geq 1$, (12) is evidently true if $d\left(y_{n-1}, y_{n}\right)>d\left(y_{n}, y_{n+2}\right)$. Hence (12) is true for all $n \in \mathbb{N}$. Hence we have

$$
\begin{aligned}
\alpha\left(x_{n+1}, x_{n+2}\right) & =\Omega\left(d\left(y_{n}, y_{n+1}\right), d\left(y_{n+1}, y_{n+2}\right), d\left(y_{n}, y_{n+1}\right), 0, d\left(y_{n}, y_{n+2}\right)\right) \\
& \leq \Omega\left(d\left(y_{n-1}, y_{n}\right), d\left(y_{n-1}, y_{n}\right), d\left(y_{n-1}, y_{n}\right), 0, d\left(y_{n}, y_{n+2}\right)\right) \\
& \leq \Omega\left(d\left(y_{n-1}, y_{n}\right), d\left(y_{n-1}, y_{n}\right), d\left(y_{n-1}, y_{n}\right), 0, \lambda_{r} d\left(y_{n-1}, y_{n}\right)\right) \\
& \leq \varphi\left(d\left(y_{n-1}, y_{n}\right)\right)
\end{aligned}
$$


for all $n \in \mathbb{N}$. Hence from (3) we have

$$
d\left(y_{n+1}, y_{n+2}\right) \leq \varphi\left(d\left(y_{n-1}, y_{n}\right)\right)
$$

for all $n \in \mathbb{N}$. Since $\varphi$ is monotonically increasing on $\mathbb{R}^{+}$, by repeatedly using inequality (14) we obtain

$$
d\left(y_{2 n}, y_{2 n+1}\right) \leq \varphi^{n}\left(d\left(y_{0}, y_{1}\right)\right), \quad d\left(y_{2 n+1}, y_{2 n+2}\right) \leq \varphi^{n}\left(d\left(y_{1}, y_{2}\right)\right)
$$

for all $n \in \mathbb{N}$. Since $\varphi$ is monotonically increasing on $\mathbb{R}^{+}$and $\varphi\left(t^{+}\right)<t$ for all $t \in$ $(0, \infty),\left\{\varphi^{n}(t)\right\}$ converges to zero for all $t$ in $\mathbb{R}^{+}$. Hence from (15) it follows that $\left\{d\left(y_{n}, y_{n+1}\right)\right\}$ converges to zero.

DEFINITION 6 (see [5]). A pair $\left(f_{1}, f_{2}\right)$ of self-maps on $(X, d)$ is said to be compatible (co.) if $\left\{d\left(f_{1} f_{2} x_{n}, f_{2} f_{1} x_{n}\right)\right\}$ converges to zero whenever $\left\{x_{n}\right\}$ is a sequence in $X$ such that $\left\{f_{1} x_{n}\right\}$ and $\left\{f_{2} x_{n}\right\}$ are convergent in $X$ and have the same limit.

DEFINITION 7 (see [4]). A pair $\left(f_{1}, f_{2}\right)$ of self-maps on an arbitrary set $E$ is said to be weakly compatible (w.co.) if $f_{1} f_{2} x=f_{2} f_{1} x$ whenever $x \in E$ is such that $f_{1} x=f_{2} x$.

REMARK 8. If $(f, S)$ is co. then it is w.co.

Definition 9 (see [7]). A pair $\left(f_{1}, f_{2}\right)$ of self-maps on $(X, d)$ is said to be reciprocally continuous on $X$ if $\left\{f_{1} f_{2} x_{n}\right\}$ converges to $f_{1} u$ and $\left\{f_{2} f_{1} x_{n}\right\}$ converges to $f_{2} u$ whenever $\left\{x_{n}\right\}$ is a sequence in $X$ such that $\left\{f_{1} x_{n}\right\}$ and $\left\{f_{2} x_{n}\right\}$ converge to $u$ for some $u \in X$.

DEFinition 10. A pair $\left(f_{1}, f_{2}\right)$ of self-maps on $(X, d)$ is said to be reciprocally continuous at $u \in X$ if $\left\{f_{1} f_{2} x_{n}\right\}$ converges to $f_{1} u$ and $\left\{f_{2} f_{1} x_{n}\right\}$ converges to $f_{2} u$ whenever $\left\{x_{n}\right\}$ is a sequence in $X$ such that $\left\{f_{1} x_{n}\right\}$ and $\left\{f_{2} x_{n}\right\}$ converge to $u$.

LEMMA 11. Suppose that $(f, S)$ has property $A,\left\{y_{n}\right\}$ converges to an element $z$ of $X$ and that (3) is true for all $x, y$ in $X$. Then the following statements are true:

(i) If $\sigma_{1}(t)<t$ for all $t \in(0, \infty)$ and $f p=S p$ for some $p \in X$, then $f p=z$. In particular, $f$ and $S$ cannot have a common fixed point or coincidence value other than $z$ if $\sigma_{1}(t)<t$ for all $t \in(0, \infty)$.

(ii) If $\sigma_{2}(t)<t$ for all $t \in(0, \infty)$ and $z \in \mathfrak{R}(S)$, then there exists $w \in X$ such that $f w=S w=z$.

(iii) If $\zeta(t)<t$ for all $t \in(0, \infty), z \in \mathfrak{R}(S)$ and $(f, S)$ is w.co., then $f z=S z=z$.

(iv) If $\sigma_{1}(t)<t$ for all $t \in(0, \infty), S$ is continuous at $z$ and $(f, S)$ is co., then $S z=z$.

(v) If $\sigma_{1}(t)<t$ for all $t \in(0, \infty), f$ is continuous at $z$ and $(f, S)$ is co., then $f z=z$.

(vi) If $\sigma_{1}(t)<t$ for all $t \in(0, \infty),(f, S)$ is co. and reciprocally continuous at $z$, then $f z=S z=z$.

Proof. (i) Suppose that $\sigma_{1}(t)<t$ for all $t \in(0, \infty)$ and $f p=S p$ for some $p \in X$. We have

$$
\alpha\left(p, x_{n+1}\right)=\Omega\left(0, d\left(y_{n}, y_{n+1}\right), d\left(f p, y_{n}\right), d\left(f p, y_{n}\right), d\left(f p, y_{n+1}\right)\right)
$$

for all $n \in \mathbb{N}$. We note that the limit superior of the sequence $\left\{\alpha\left(p, x_{n+1}\right)\right\}$ is less than 
or equal to $\Omega\left(0,0^{+}, d(f p, z)^{+}, d(f p, z)^{+}, d(f p, z)^{+}\right)$which in turn is less than or equal to $\sigma_{1}(d(f p, z))$. From (3) we have

$$
d\left(f p, y_{n+1}\right) \leq \alpha\left(p, x_{n+1}\right)
$$

for all $n \in \mathbb{N}$. By taking limit superiors on both sides of (17) as $n \rightarrow+\infty$ we obtain $d(f p, z) \leq \sigma_{1}(d(f p, z))$. Since $\sigma_{1}(t)<t$ for all $t \in(0, \infty)$, we have $d(f p, z)=0$. Hence $f p=z$. Hence $f$ and $S$ cannot have a common fixed point other than $z$. If $p, q \in X$ are such that $f p=S p$ and $f q=S q$, then we have $f p=z=f q$. Hence $f$ and $S$ cannot have a coincidence value other than $z$.

(ii) Suppose that $\sigma_{2}(t)<t$ for all $t \in(0, \infty)$ and $z \in \mathfrak{R}(S)$. Then there exists $w \in$ $X \ni S w=z$. We have

$$
\alpha\left(w, x_{n+1}\right)=\Omega\left(d(z, f w), d\left(y_{n}, y_{n+1}\right), d\left(z, y_{n}\right), d\left(f w, y_{n}\right), d\left(z, y_{n+1}\right)\right)
$$

for all $n \in \mathbb{N}$. We note that the limit superior of the sequence $\left\{\alpha\left(w, x_{n+1}\right)\right\}$ is less than or equal to $\Omega\left(d(f w, z), 0^{+}, 0^{+}, d(f w, z)^{+}, 0^{+}\right)$which in turn is less than or equal to $\sigma_{2}(d(f w, z))$. From (3) we have

$$
d\left(f w, y_{n+1}\right) \leq \alpha\left(w, x_{n+1}\right)
$$

for all $n \in \mathbb{N}$. By taking limit superiors on both sides of (19) as $n \rightarrow+\infty$ we obtain $d(f w, z) \leq \sigma_{2}(d(f w, z))$. Hence $d(f w, z)=0$. Hence $f w=z$.

(iii) Suppose that $\zeta(t)<t$ for all $t \in(0, \infty), z \in \mathfrak{R}(S)$ and $(f, S)$ is w.co. From statement (ii) it follows that there exists $w \in X \ni f w=S w=z$. Hence from the weak compatibility of $(f, S)$ we have $f z=f S w=S f w=S z$. We have

$$
\alpha\left(z, x_{n+1}\right)=\Omega\left(d(S z, f z), d\left(y_{n}, y_{n+1}\right), d\left(S z, y_{n}\right), d\left(f z, y_{n}\right), d\left(S z, y_{n+1}\right)\right)
$$

for all $n \in \mathbb{N}$. We note that the limit superior of the sequence $\left\{\alpha\left(z, x_{n+1}\right)\right\}$ is less than or equal to $\Omega\left(0,0^{+}, d(f z, z)^{+}, d(f z, z)^{+}, d(f z, z)^{+}\right)$which in turn is less than or equal to $\sigma_{1}(d(f z, z))$. From (3) we have

$$
d\left(f z, y_{n+1}\right) \leq \alpha\left(z, x_{n+1}\right)
$$

for all $n \in \mathbb{N}$. By taking limit superiors on both sides of (21) as $n \rightarrow+\infty$ we obtain $d(f z, z) \leq \sigma_{1}(d(f z, z))$. Since $\sigma_{1}(t)<t$ for all $t \in(0, \infty)$, we have $d(f z, z)=0$. Hence $f z=z$. Hence $S z=z$.

(iv) Suppose that $\sigma_{1}(t)<t$ for all $t \in(0, \infty), S$ is continuous at $z$ and $(f, S)$ is co. Since $\left\{y_{n}\right\}$ converges to $z$ and $S$ is continuous at $z,\left\{S y_{n}\right\}$ converges to $S z$. Hence the sequences $\left\{S S x_{n}\right\}$ and $\left\{S f x_{n}\right\}$ converge to $S z$. Since $(f, S)$ is co., and $\left\{f x_{n}\right\}$ and $\left\{S x_{n}\right\}$ are convergent sequences having the same limit $z$, it follows that $\left\{d\left(S f x_{n}, f S x_{n}\right)\right\}$ converges to zero. Since $\left\{S f x_{n}\right\}$ converges to $S z$, it follows that $\left\{f S x_{n}\right\}$ also converges to $S z$. We have

$$
\begin{gathered}
\alpha\left(S x_{n}, x_{n+1}\right)=\Omega\left(d\left(S S x_{n}, f S x_{n}\right), d\left(y_{n}, y_{n+1}\right), d\left(S S x_{n}, y_{n}\right),\right. \\
\left.d\left(f S x_{n}, y_{n}\right), d\left(S S x_{n}, y_{n+1}\right)\right)
\end{gathered}
$$


for all $n \in \mathbb{N}$. We note that the limit superior of the sequence $\left\{\alpha\left(S x_{n}, x_{n+1}\right)\right\}$ is less than or equal to $\Omega\left(0^{+}, 0^{+}, d(S z, z)^{+}, d(S z, z)^{+}, d(S z, z)^{+}\right)=\sigma_{1}(d(S z, z))$. From (3) we have

$$
d\left(f S x_{n}, y_{n+1}\right) \leq \alpha\left(S x_{n}, x_{n+1}\right)
$$

for all $n \in \mathbb{N}$. By taking limit superiors on both sides of (23) as $n \rightarrow+\infty$ we obtain $d(S z, z) \leq \sigma_{1}(d(S z, z))$. Hence $d(S z, z)=0$. Hence $S z=z$.

(v) Suppose that $\sigma_{1}(t)<t$ for all $t \in(0, \infty), f$ is continuous at $z$ and $(f, S)$ is co. Since $\left\{y_{n}\right\}$ converges to $z$ and $f$ is continuous at $z$, $\left\{f y_{n}\right\}$ converges to $f z$. Hence the sequences $\left\{f f x_{n}\right\}$ and $\left\{f S x_{n}\right\}$ converge to $f z$. Since $(f, S)$ is co., and $\left\{f x_{n}\right\}$ and $\left\{S x_{n}\right\}$ are convergent sequences having the same limit $z$, it follows that $\left\{d\left(S f x_{n}, f S x_{n}\right)\right\}$ converges to zero. Since $\left\{f S x_{n}\right\}$ converges to $f z$, it follows that $\left\{S f x_{n}\right\}$ also converges to $f z$. We have

$$
\begin{gathered}
\alpha\left(f x_{n}, x_{n+1}\right)=\Omega\left(d\left(S f x_{n}, f f x_{n}\right), d\left(y_{n}, y_{n+1}\right), d\left(S f x_{n}, y_{n}\right),\right. \\
\left.d\left(f f x_{n}, y_{n}\right), d\left(S f x_{n}, y_{n+1}\right)\right)
\end{gathered}
$$

for all $n \in \mathbb{N}$. We note that the limit superior of the sequence $\left\{\alpha\left(f x_{n}, x_{n+1}\right)\right\}$ is less than or equal to $\Omega\left(0^{+}, 0^{+}, d(f z, z)^{+}, d(f z, z)^{+}, d(f z, z)^{+}\right)=\sigma_{1}(d(f z, z))$. From (3) we have

$$
d\left(f f x_{n}, y_{n+1}\right) \leq \alpha\left(f x_{n}, x_{n+1}\right)
$$

for all $n \in \mathbb{N}$. By taking limit superiors on both sides of (25) as $n \rightarrow+\infty$ we obtain $d(f z, z) \leq \sigma_{1}(d(f z, z))$. Hence $d(f z, z)=0$. Hence $f z=z$.

(vi) Suppose that $\sigma_{1}(t)<t$ for all $t \in(0, \infty)$, and $(f, S)$ is co. and reciprocally continuous at $z$. Since $\left\{y_{n}\right\}$ converges to $z$, the sequences $\left\{f x_{n}\right\}$ and $\left\{S x_{n}\right\}$ are convergent and have the same limit $z$. Hence from the reciprocal continuity of $(f, S)$ at $z$ it follows that $\left\{S f x_{n}\right\}$ converges to $S z$ and $\left\{f S x_{n}\right\}$ converges to $f z$ and from the compatibility of $(f, S)$ it follows that $\left\{d\left(S f x_{n}, f S x_{n}\right)\right\}$ converges to zero. Hence $f z=S z$. We have

$$
\alpha\left(z, x_{n+1}\right)=\Omega\left(d(S z, f z), d\left(y_{n}, y_{n+1}\right), d\left(S z, y_{n}\right), d\left(f z, y_{n}\right), d\left(S z, y_{n+1}\right)\right)
$$

for all $n \in \mathbb{N}$. We note that the limit superior of the sequence $\left\{\alpha\left(z, x_{n+1}\right)\right\}$ is less than or equal to $\Omega\left(0,0^{+}, d(f z, z)^{+}, d(f z, z)^{+}, d(f z, z)^{+}\right)$which in turn is less than or equal to $\sigma_{1}(d(f z, z))$. From (3) we have

$$
d\left(f z, y_{n+1}\right) \leq \alpha\left(z, x_{n+1}\right)
$$

for all $n \in \mathbb{N}$. By taking limit superiors on both sides of the above inequality as $n \rightarrow+\infty$ we obtain $d(f z, z) \leq \sigma_{1}(d(f z, z))$. Hence $d(f z, z)=0$. Hence $f z=z$. Hence $S z=z$.

THEOREM 12. Suppose that $(f, S)$ has property $A, \Omega$ has properties $A$ and $B, \sigma_{1}(t)<$ $t$ for all $t \in(0, \infty)$ and that inequality (3) is true for all $x, y$ in $X$. Then $\left\{y_{n}\right\}$ is Cauchy. Suppose that it converges to an element $z$ of $X$. Then the following statements are true:

(i) $f$ and $S$ cannot have a common fixed point or coincidence value other than $z$.

(ii) If $S$ is continuous at $z$ and $(f, S)$ is co., then $S z=z$.

(iii) If $f$ is continuous at $z$ and $(f, S)$ is co., then $f z=z$.

(iv) If $(f, S)$ is co. and reciprocally continuous at $z$, then $f z=S z=z$. 
(v) If $\sigma_{2}(t)<t$ for all $t \in(0, \infty)$ and $z \in \mathfrak{R}(S)$, then there exists $w \in X$ such that $f w=S w=z$.

(vi) If $\sigma_{2}(t)<t$ for all $t \in(0, \infty), z \in \mathfrak{R}(S)$ and $(f, S)$ is w.co., then $f z=S z=z$.

Proof. The proof follows from Lemmas 2, 5, and 11.

COROLLARY 13. Suppose that $(f, S)$ has property $A$ and that there is a monotonically decreasing function $\delta: \mathbb{R}^{+} \rightarrow(0,1 / 3]$ such that

$$
\begin{aligned}
d(f x, f y) \leq \sup \{ & \operatorname{ad}(S x, S y)+b \max \{d(S x, f x), d(S y, f y)\} \\
+ & c[d(f x, S y)+d(S x, f y)]: a \geq 0, b \geq \delta(\theta(x, y)), \\
c & \geq \delta(\theta(x, y)), a+b+2 c \leq 1\}
\end{aligned}
$$

for all $x, y$ in $X$, where

$$
\theta(x, y)=\max \left\{d(S x, S y), d(S x, f x), d(S y, f y), \frac{1}{2}[d(f x, S y)+d(S x, f y)]\right\} .
$$

Then $\left\{y_{n}\right\}$ is Cauchy. Suppose that it converges to an element $z$ of $X$. Then the following statements are true:

(i) $f$ and $S$ cannot have a common fixed point or coincidence value other than $z$.

(ii) If $z \in \mathfrak{R}(S)$, then there exists $w \in X$ such that $f w=S w=z$.

(iii) If $z \in \mathfrak{R}(S)$ and $(f, S)$ is w.co., then $f z=S z=z$.

(iv) If $S$ is continuous at $z$ and $(f, S)$ is co., then $f z=S z=z$.

(v) If $f$ is continuous at $z$ and $(f, S)$ is co., then $f z=z$.

(vi) If $(f, S)$ is co. and reciprocally continuous at $z$, then $f z=S z=z$.

Proof. Define $\Lambda:\left(\mathbb{R}^{+}\right)^{5} \rightarrow \mathbb{R}^{+}$as $\Lambda\left(t_{1}, t_{2}, t_{3}, t_{4}, t_{5}\right)=\max \left\{t_{1}, t_{2}, t_{3},(1 / 2)\left(t_{4}+t_{5}\right)\right\}$. Define $I: \mathbb{R}^{+} \rightarrow \mathbb{R}^{+}$as $I(t)=\left\{(a, b, c) \in\left(\mathbb{R}^{+}\right)^{3}: a \geq 0, b \geq \delta(t), c \geq \delta(t)\right.$, and $a+b+$ $2 c \leq 1\}$. Define $\Omega:\left(\mathbb{R}^{+}\right)^{5} \rightarrow \mathbb{R}^{+}$as

$$
\begin{aligned}
& \Omega\left(t_{1}, t_{2}, t_{3}, t_{4}, t_{5}\right) \\
& \quad=\sup \left\{a t_{3}+b \max \left\{t_{1}, t_{2}\right\}+c\left(t_{4}+t_{5}\right):(a, b, c) \in I\left(\Lambda\left(t_{1}, t_{2}, t_{3}, t_{4}, t_{5}\right)\right)\right\}
\end{aligned}
$$

for all $t_{1}, t_{2}, t_{3}, t_{4}, t_{5} \in \mathbb{R}^{+}$. It is clear that (3) is true for all $x, y$ in $X$. Since $\delta$ is monotonically decreasing on $\mathbb{R}^{+}$, it is evident that $\Omega$ is increasing in each coordinate variable. It can be verified that $\sigma_{1}(t) \leq\left[1-\delta\left(t^{+}\right)\right] t$ and $\sigma_{2}(t) \leq[1-\delta(t)] t$ for all $t \in(0, \infty)$ so that $\zeta(t)<t$ for all $t \in(0, \infty)$. It can be seen that $\Omega(t, s, t, 0, t+$ $s) \leq t+[1-\delta(s)](s-t)<s$ if $0 \leq t<s<+\infty$. Hence $\Omega$ has property $A$. It can be shown that $\Omega(t, t, 2 t, t, t+s) \leq \sup \{(1+a) t+c s:(a, b, c) \in I(2 t)\}$ for all $t(\mathbb{R})^{+}$ and $s \in[t, 2 t],(1+a) /(1-c) \leq 2-b \leq 2-\delta(2 t)$ for all $(a, b, c) \in I(2 t)$ and that $\Omega\left(t, t, t, 0, \lambda_{t} t\right) \leq\left[1-\left(2-\lambda_{t}\right) \delta(t)\right] t \leq[1-\delta(2 t) \delta(t)] t<t$ for all $t \in(0,+\infty)$, where $\lambda_{t}=\sup \{(1+a) /(1-c):(a, b, c) \in I(2 t)\} \leq 2-\delta(2 t)$. Clearly, $\sup \{c:(a, b, c) \in$ $I(2 t)\} \leq(1 / 2)[1-\delta(2 t)] \leq 1 / 2$. Define $\varphi: \mathbb{R}^{+} \rightarrow \mathbb{R}^{+}$as $\varphi(t)=[1-\delta(2 t) \delta(t)] t$ for all $t \in \mathbb{R}^{+}$. Since $\delta$ is monotonically decreasing on $\mathbb{R}^{+}, \varphi$ is monotonically increasing on $\mathbb{R}^{+}$. Clearly, $\varphi\left(t^{+}\right)<t$ for all $t \in(0,+\infty)$ and $\Omega\left(t, t, t, 0, \lambda_{t} t\right) \leq \varphi(t)$ for all $t \in \mathbb{R}^{+}$. Hence $\Omega$ satisfies property $B$ with $I_{t}=I(2 t), \beta_{i}=a$, and $\gamma_{i}=c$, where $i=(a, b, c) \in I(2 t)$. Now the corollary is evident from Theorem 12 . 
COROLlary 14 (see [2, Theorem 2.1]). Suppose that $f(X) \subseteq S(X)$ and that there are nonnegative real-valued functions $a, b, c$ on $X \times X$ such that

$$
\begin{aligned}
d(f x, f y) \leq & a(x, y) d(S x, S y)+b(x, y) \max \{d(S x, f x), d(S y, f y)\} \\
& +c(x, y)[d(f x, S y)+d(S x, f y)]
\end{aligned}
$$

for all $x, y$ in $X, \inf \{b(u, v): u, v \in X\}>0, \inf \{c(u, v): u, v \in X\}>0$, and $\sup \{a(u, v)$ $+b(u, v)+2 c(u, v): u, v \in X\}=1$. Suppose also that either (a) $X$ is complete and $S$ is surjective; or (b) $X$ is complete, $S$ is continuous and $(f, S)$ is co.; or (c) $S(X)$ is complete: or (d) $f(X)$ is complete. Then $f$ and $S$ have a coincidence point in $X$. Further, the coincidence value is unique.

Proof. The proof follows from Corollary 13 by taking $\delta$ as a constant function on $\mathbb{R}^{+}$with its value in $\left(0, \min \left\{1 / 3, \delta_{1}, \delta_{2}\right\}\right]$, where $\delta_{1}=\inf \{b(u, v): u, v \in X\}$ and $\delta_{2}=\inf \{c(u, v): u, v \in X\}$.

COROLlARY 15. Suppose that $(f, S)$ has property $A$ and that there are a positive integer $N$, nonnegative constants $a_{1}, \ldots, a_{N}$, and positive constants $b_{1}, \ldots, b_{N}, c_{1}, \ldots, c_{N}$ such that $a_{i}+b_{i}+2 c_{i} \leq 1$ for all $i=1,2, \ldots, N$ and

$$
\begin{array}{r}
d(f x, f y) \leq \max \left\{a_{i} d(S x, S y)+b_{i} \max \{d(S x, f x), d(S y, f y)\}\right. \\
\left.+c_{i}[d(f x, S y)+d(S x, f y)]: i \in\{1,2, \ldots, N\}\right\}
\end{array}
$$

for all $x, y$ in $X$. Then $\left\{y_{n}\right\}$ is Cauchy. Suppose that it converges to an element $z$ of $X$. Then statements (i) to (vi) of Corollary 13 are true here also.

Proof. The proof follows from Corollary 13 by taking $\delta$ as a constant function on $\mathbb{R}^{+}$with its value in $\left(0, \min \left\{1 / 3, \delta_{1}, \delta_{2}\right\}\right]$, where $\delta_{1}=\min \left\{b_{k}: k=1,2, \ldots, N\right\}$ and $\delta_{2}=\min \left\{c_{k}: k=1,2, \ldots, N\right\}$.

Corollary 16 (see uniqueness part of [2, Theorem 2.1]). Suppose that $(X, d)$ is complete and

$$
\begin{aligned}
d(f x, f y) \leq & a(\max \{d(x, y), d(x, f x), d(y, f y),[d(f x, y)+d(x, f y)] / 2\}) \\
& +b(\max \{d(x, f x), d(y, f y)\})+c[d(f x, y)+d(x, f y)]
\end{aligned}
$$

for all $x, y$ in $X$ and for some constants $a, b, c$ with $a \geq 0, b>0, c>0$ and $a+b+2 c=1$. Then $f$ has a unique fixed point in $X$.

Proof. The proof follows from Corollary 15 by taking $S=I, N=3, a_{1}=a, b_{1}=$ $b_{3}=b, c_{1}=c_{2}=c, a_{2}=a_{3}=0, b_{2}=a+b, c_{3}=(1 / 2) a+c$.

COROLlary 17. Let $\varphi: \mathbb{R}^{+} \rightarrow \mathbb{R}^{+}$be a monotonically increasing map such that $\varphi\left(t^{+}\right)<t$ for all $t \in(0, \infty)$. Suppose that $(f, S)$ has property $A$ and

$$
\begin{aligned}
& d(f x, f y) \\
& \quad \leq \varphi\left(\max \left\{d(S x, S y), d(S x, f x), d(S y, f y), \frac{1}{2}[d(f x, S y)+d(S x, f y)]\right\}\right)
\end{aligned}
$$


for all $x, y$ in $X$. Then $\left\{y_{n}\right\}$ is Cauchy. Suppose that it converges to an element $z$ of $X$. Then statements (i) to (vi) of Corollary 13 are true here also.

Proof. Define $\Omega:\left(\mathbb{R}^{+}\right)^{5} \rightarrow \mathbb{R}^{+}$as

$$
\Omega\left(t_{1}, t_{2}, t_{3}, t_{4}, t_{5}\right)=\varphi\left(\max \left\{t_{1}, t_{2}, t_{3}, \frac{1}{2}\left(t_{4}+t_{5}\right)\right\}\right)
$$

for all $t_{1}, t_{2}, t_{3}, t_{4}, t_{5} \in \mathbb{R}^{+}$. It is clear that $\Omega$ is increasing in each coordinate variable, $\zeta(t)<t$ for all $t \in(0, \infty), \Omega$ has property $A$ and that (3) is true for all $x, y$ in $X$. Clearly, $\Omega(t, t, 2 t, t, t+s)=\varphi(2 t) \leq 2 t$ if $t \in \mathbb{R}^{+}$and $t \leq s \leq 2 t$. Also $\Omega(t, t, t, 0,2 t)=\varphi(t)$ for all $t \in \mathbb{R}^{+}$. Hence $\Omega$ satisfies property $B$ with $I_{t}$ being a singleton, $\beta_{i}=1, \gamma_{i}=0\left(i \in I_{t}\right)$ and $\lambda_{t}=2$ for all $t \in \mathbb{R}^{+}$. Now the corollary is evident from Theorem 12 .

REMARK 18. Example 19 shows that Corollary 17 cannot be deduced from Corollary 13 and therefore from Corollary 14 also. In particular, it follows that Theorem 12 is a proper generalization of [2, Theorem 2.1].

EXAMPLE 19. Define $f:[0,1] \rightarrow[0,1]$ as $f(x)=x /(1+x)$ for all $x \in[0,1]$ and $\varphi$ : $\mathbb{R}^{+} \rightarrow \mathbb{R}^{+}$as $\varphi(t)=t /(1+t)$ for all $t \in \mathbb{R}^{+}$. Then $\varphi$ is a strictly increasing continuous function on $\mathbb{R}^{+}, \varphi(t)<t$ for all $t \in(0, \infty)$ and $|f x-f y| \leq \varphi(|x-y|)$ for all $x, y$ in $X$. For $x, y \in[0,1]$ let $\theta(x, y)=\max \{|x-y|, \max \{|x-f x|,|y-f y|\},(1 / 2)[|f x-y|+$ $|x-f y|]\}$. Let $\delta: \mathbb{R}^{+} \rightarrow(0,1 / 3]$ be a monotonically decreasing function. For $t \in \mathbb{R}^{+}$ let $I(t)=\left\{(a, b, c) \in\left(\mathbb{R}^{+}\right)^{3}: a \geq 0, b \geq \delta(t), c \geq \delta(t)\right.$ and $\left.a+b+2 c \leq 1\right\}$. If possible, suppose that

$$
\begin{aligned}
|f x-f y| \leq \sup \{a|x-y|+b \max \{|x-f x|,|y-f y|\} \\
+c[|f x-y|+|x-f y|]:(a, b, c) \in I(\theta(x, y))\}
\end{aligned}
$$

for all $x, y$ in $[0,1]$. Then, since $I(1) \supseteq I(\theta(x, y))$ for all $x, y$ in $[0,1]$, we have

$$
\begin{aligned}
|f x-f y| \leq \sup \{a|x-y|+b \max \{|x-f x|,|y-f y|\} \\
+c[|f x-y|+|x-f y|]:(a, b, c) \in I(1)\}
\end{aligned}
$$

for all $x, y$ in $[0,1]$. Hence for $x \in(0,(1 / 2) \delta(1)]$ and $y=0$ we have

$$
\frac{x}{1+x} \leq \sup \left\{a x+b \frac{x^{2}}{1+x}+c\left[\frac{x}{1+x}+x\right]:(a, b, c) \in I(1)\right\}
$$

so that we have

$$
\begin{aligned}
1 & \leq \sup \{a(1+x)+b x+c(2+x):(a, b, c) \in I(1)\} \\
& =\sup \{a+2 c+x(a+b+c):(a, b, c) \in I(1)\} \\
& \leq \sup \{a+2 c+x:(a, b, c) \in I(1)\} \\
& \leq \sup \left\{a+2 c+\frac{1}{2} \delta(1):(a, b, c) \in I(1)\right\} \\
& \leq \sup \{a+b+2 c:(a, b, c) \in I(1)\}-\frac{1}{2} \delta(1) \\
& =1-\frac{1}{2} \delta(1)<1
\end{aligned}
$$

which is a contradiction. 
DEFINITION 20. We say that $\Omega$ has property $C$ if there exist (i) a monotonically increasing function $\varphi: \mathbb{R}^{+} \rightarrow \mathbb{R}^{+}$with $\sum_{n=1}^{\infty} \varphi^{n}(t)<+\infty$ for all $t \in \mathbb{R}^{+}$, and (ii) for each $t \in \mathbb{R}^{+}$a nonempty index set $I_{t}$ and nonnegative real numbers $\beta_{i}, \gamma_{i}\left(i \in I_{t}\right)$ such that $\sup \left\{\gamma_{i}: i \in I_{t}\right\}<1, \Omega(t, t, 2 t, t, t+s) \leq \sup \left\{\left(1+\beta_{i}\right) t+\gamma_{i} s: i \in I_{t}\right\}$ for all $s \in[t, 2 t]$, $\Omega\left(t, t, t, 0, \lambda_{t} t\right) \leq \varphi(t)$, where $\lambda_{t}=\sup \left\{\left(1+\beta_{i}\right) /\left(1-\gamma_{i}\right): i \in I_{t}\right\}$.

Definition 21. We say that $(F, S)$ has property $A$ if there is a sequence $\left\{u_{n}\right\}_{n=0}^{\infty}$ in $X$ such that $S u_{n+1} \in F u_{n}$ and $d\left(S u_{n}, F u_{n}\right)=d\left(S u_{n}, S u_{n+1}\right)$ for all $n=0,1,2 \ldots$ (Let $v_{n}$ stand for $S u_{n+1}$.)

LEMmA 22. Suppose that $(F, S)$ has property $A, \Omega$ has properties $A$ and $C$, and

$$
H(F x, F y) \leq \Omega(d(S x, F x), d(S y, F y), d(S x, S y), d(S y, F x), d(S x, F y))
$$

for all $x, y$ in $X$. Then $\left\{v_{n}\right\}_{n=1}^{\infty}$ is Cauchy.

Proof. We have $D\left(u_{n}, u_{n+1}\right) \leq \Omega\left(d\left(v_{n-1}, v_{n}\right), d\left(v_{n}, v_{n+1}\right), d\left(v_{n-1}, v_{n}\right), 0, d\left(v_{n-1}\right.\right.$, $\left.v_{n+1}\right)$ ) for all $n \in \mathbb{N}$ since $\Omega$ is increasing in each coordinate variable. From (40) we have

$$
d\left(v_{n}, v_{n+1}\right)=d\left(S u_{n+1}, F u_{n+1}\right) \leq H\left(F u_{n}, F u_{n+1}\right) \leq D\left(u_{n}, u_{n+1}\right)
$$

for all $n \in \mathbb{N}$. Now proceeding as in the proof of Lemma 5 it can be seen that $d\left(v_{n}, v_{n+1}\right)$ $\leq d\left(v_{n-1}, v_{n}\right)$ for all $n \in \mathbb{N}$. From (40) we have

$$
d\left(v_{n}, F u_{n+2}\right)=d\left(S u_{n+1}, F u_{n+2}\right) \leq H\left(F u_{n}, F u_{n+2}\right) \leq D\left(u_{n}, u_{n+2}\right)
$$

for all $n \in \mathbb{N}$. But

$$
\begin{aligned}
D\left(u_{n}, u_{n+2}\right) \leq & \Omega\left(d\left(v_{n-1}, v_{n}\right), d\left(v_{n+1}, v_{n+2}\right), d\left(v_{n-1}, v_{n+1}\right),\right. \\
& \left.d\left(v_{n}, v_{n+1}\right), d\left(v_{n-1}, F u_{n+2}\right)\right) \\
\leq & \Omega\left(d\left(v_{n-1}, v_{n}\right), d\left(v_{n+1}, v_{n+2}\right), d\left(v_{n-1}, v_{n}\right)+d\left(v_{n}, v_{n+1}\right),\right. \\
& \left.d\left(v_{n}, v_{n+1}\right), d\left(v_{n-1}, v_{n}\right)+d\left(v_{n}, F u_{n+2}\right)\right) \\
\leq & \Omega\left(d\left(v_{n-1}, v_{n}\right), d\left(v_{n-1}, v_{n}\right), 2 d\left(v_{n-1}, v_{n}\right), d\left(v_{n-1}, v_{n}\right),\right. \\
& \left.d\left(v_{n-1}, v_{n}\right)+d\left(v_{n}, F u_{n+2}\right)\right)
\end{aligned}
$$

since the sequence $\left\{d\left(v_{k-1}, v_{k}\right)\right\}$ is monotonically decreasing and $\Omega$ is increasing in each coordinate variable. Since $\Omega$ has property $C$, there exist (i) a monotonically increasing function $\varphi: \mathbb{R}^{+} \rightarrow \mathbb{R}^{+}$with $\sum_{n=1}^{\infty} \varphi^{n}(t)<+\infty$ for all $t \in \mathbb{R}^{+}$and (ii) for each $t \in \mathbb{R}^{+}$a nonempty index set $I_{t}$ and nonnegative real numbers $\beta_{i}, \gamma_{i}\left(i \in I_{t}\right)$ such that $\sup \left\{\gamma_{i}: i \in I_{t}\right\}<1, \Omega(t, t, 2 t, t, t+s) \leq \sup \left\{\left(1+\beta_{i}\right) t+\gamma_{i} s: i \in I_{t}\right\}$ for all $s \in[t, 2 t]$, and $\Omega\left(t, t, t, 0, \lambda_{t} t\right) \leq \varphi(t)$, where $\lambda_{t}=\sup \left\{\left(1+\beta_{i}\right) /\left(1-\gamma_{i}\right): i \in I_{t}\right\}$. Since $d\left(v_{n}, F u_{n+2}\right) \leq d\left(v_{n}, v_{n+2}\right) \leq d\left(v_{n}, v_{n+1}\right)+d\left(v_{n+1}, v_{n+2}\right) \leq 2 d\left(v_{n-1}, v_{n}\right)$, we have

$$
\begin{aligned}
& \Omega\left(d\left(v_{n-1}, v_{n}\right), d\left(v_{n-1}, v_{n}\right), 2 d\left(v_{n-1}, v_{n}\right), d\left(v_{n-1}, v_{n}\right), d\left(v_{n-1}, v_{n}\right)+d\left(v_{n}, F u_{n+2}\right)\right) \\
& \quad \leq \sup \left\{\left(1+\beta_{i}\right) d\left(v_{n-1}, v_{n}\right)+\gamma_{i} d\left(v_{n}, F u_{n+2}\right): i \in I_{r}\right\}
\end{aligned}
$$


provided $d\left(v_{n-1}, v_{n}\right) \leq d\left(v_{n}, F u_{n+2}\right)$, where $r=d\left(v_{n-1}, v_{n}\right)$. Hence from (42), (43), and (44) we have

$$
d\left(v_{n}, F u_{n+2}\right) \leq \sup \left\{\left(1+\beta_{i}\right) d\left(v_{n-1}, v_{n}\right)+\gamma_{i} d\left(v_{n}, F u_{n+2}\right): i \in I_{r}\right\}
$$

provided $d\left(v_{n-1}, v_{n}\right) \leq d\left(v_{n}, F u_{n+2}\right)$. Now proceeding as in the proof of Lemma 5 , it can be shown that

$$
d\left(v_{n}, F u_{n+2}\right) \leq \lambda_{r} d\left(v_{n-1}, v_{n}\right)
$$

for all $n \in \mathbb{N}$. Hence we have

$$
\begin{aligned}
D\left(u_{n+1}, u_{n+2}\right) & =\Omega\left(d\left(v_{n}, v_{n+1}\right), d\left(v_{n+1}, v_{n+2}\right), d\left(v_{n}, v_{n+1}\right), 0, d\left(v_{n}, F u_{n+2}\right)\right) \\
& \leq \Omega\left(d\left(v_{n-1}, v_{n}\right), d\left(v_{n-1}, v_{n}\right), d\left(v_{n-1}, v_{n}\right), 0, d\left(v_{n}, F u_{n+2}\right)\right) \\
& \leq \Omega\left(d\left(v_{n-1}, v_{n}\right), d\left(v_{n-1}, v_{n}\right), d\left(v_{n-1}, v_{n}\right), 0, \lambda_{r} d\left(v_{n-1}, v_{n}\right)\right) \\
& \leq \varphi\left(d\left(v_{n-1}, v_{n}\right)\right)
\end{aligned}
$$

for all $n \in \mathbb{N}$. Hence from (40) we have

$$
\begin{aligned}
d\left(v_{n+1}, v_{n+2}\right) & =d\left(S u_{n+2}, F u_{n+2}\right) \\
& \leq H\left(F u_{n+1}, F u_{n+2}\right) \leq D\left(u_{n+1}, u_{n+2}\right) \leq \varphi\left(d\left(v_{n-1}, v_{n}\right)\right)
\end{aligned}
$$

that is,

$$
d\left(v_{n+1}, v_{n+2}\right) \leq \varphi\left(d\left(v_{n-1}, v_{n}\right)\right)
$$

for all $n \in \mathbb{N}$. Since $\varphi$ is monotonically increasing on $\mathbb{R}^{+}$, by repeatedly using (49) we obtain

$$
d\left(v_{2 n}, v_{2 n+1}\right) \leq \varphi^{n}\left(d\left(v_{0}, v_{1}\right)\right), \quad d\left(v_{2 n+1}, v_{2 n+2}\right) \leq \varphi^{n}\left(d\left(v_{1}, v_{2}\right)\right),
$$

for all $n \in \mathbb{N}$. Since $\sum_{n=1}^{\infty} \varphi^{n}(t)<+\infty$ for all $t \in \mathbb{R}^{+}$, from (50) it follows that $\sum_{n=1}^{\infty} d\left(v_{n}\right.$, $v_{n+1}$ ) is convergent. Hence $\left\{v_{n}\right\}_{n=1}^{\infty}$ is Cauchy.

THEOREM 23. Suppose that $(F, S)$ has property $A, \Omega$ has properties $A$ and $C$ and that (40) is true for all $x, y$ in $X$. Then $\left\{v_{n}\right\}$ is Cauchy. Suppose that it converges to an element $z$ of $S(X)$ and $\sigma_{2}(t)<t$ for all $t \in(0, \infty)$. Then $S w \in F w$ for any $w \in X \ni S w=z$.

Proof. The proof that $\left\{v_{n}\right\}$ is Cauchy follows from Lemma 22. Suppose that it converges to an element $z$ of $S(X)$. Let $w \in X$ be such that $z=S w$. We have

$$
D\left(w, u_{n+1}\right)=\Omega\left(d(z, F w), d\left(v_{n}, v_{n+1}\right), d\left(z, v_{n}\right), d\left(v_{n}, F w\right), d\left(z, F u_{n+1}\right)\right)
$$

for all $n \in \mathbb{N}$. We note that the limit superior of the sequence $\left\{D\left(w, u_{n+1}\right)\right\}$ is less than or equal to $\Omega\left(d(z, F w), 0^{+}, 0^{+}, d(z, F w)^{+}, 0^{+}\right)$which in turn is less than or equal to $\sigma_{2}(d(z, F w))$. From (40) we have

$$
d\left(v_{n+1}, F w\right) \leq H\left(F w, F u_{n+1}\right) \leq D\left(w, u_{n+1}\right)
$$


for all $n \in \mathbb{N}$. By taking limit superiors on both sides of (52) as $n \rightarrow+\infty$ we obtain $d(z, F w) \leq \sigma_{2}(d(z, F w))$. Since $\sigma_{2}(t)<t$ for all $t \in(0, \infty)$, we have $d(z, F w)=0$. Since $F w$ is closed, $z \in F w$.

COROLlary 24 (see [2, Theorem 2.2]). Suppose that $F(x) \in K(X)$ for all $x \in X$, $F(x) \subseteq S(X)$ for all $x \in X$ and that there are nonnegative real-valued functions $a, b, c$ on $X \times X$ such that

$$
\begin{aligned}
H(F x, F y) \leq & a(x, y) d(S x, S y)+b(x, y) \max \{d(S x, F x), d(S y, F y)\} \\
& +c(x, y)[d(S y, F x)+d(S x, F y)]
\end{aligned}
$$

for all $x, y$ in $X, \inf \{b(u, v): u, v \in X\}>0, \inf \{c(u, v): u, v \in X\}>0$, and $\sup \{a(u, v)+b(u, v)+2 c(u, v): u, v \in X\}=1$. Suppose also that either (a) $X$ is $(F, S)$ orbitally complete and $S$ is surjective; or (b) $S(X)$ is $(F, S)$ orbitally complete: or (c) $F(X)$ is $(F, S)$ orbitally complete. Then $F$ and $S$ have a coincidence point in $X$.

Proof. Let $\delta=\min \left\{1 / 3, \delta_{1}, \delta_{2}\right\}$, where $\delta_{1}=\inf \{b(u, v): u, v \in X\}$ and $\delta_{2}=$ $\inf \{c(u, v): u, v \in X\}$. Define $\Omega:\left(\mathbb{R}^{+}\right)^{5} \rightarrow \mathbb{R}^{+}$as

$$
\begin{aligned}
& \Omega\left(t_{1}, t_{2}, t_{3}, t_{4}, t_{5}\right)=\sup \left\{a t_{3}+b \max \left\{t_{1}, t_{2}\right\}+c\left(t_{4}+t_{5}\right):\right. \\
& a \geq 0, b \geq \delta, c \geq \delta, \text { and } a+b+2 c \leq 1\} .
\end{aligned}
$$

As in the proof of Corollary 13 it can be seen that $\Omega$ has properties $A$ and $C$ and that $\sigma_{2}(t)<t$ for all $t \in(0, \infty)$. Evidently (40) is true for all $x, y$ in $X$. Since the values of $F$ are compact and $F x \subseteq S(X)$ for all $x$ in $X,(F, S)$ has property $A$. Now the corollary is evident from Theorem 23.

COROLlary 25. Let $\varphi: \mathbb{R}^{+} \rightarrow \mathbb{R}^{+}$be a monotonically increasing map such that $\sum_{n=1}^{\infty} \varphi^{n}(t)<+\infty$ for all $t \in(0, \infty)$. Suppose that $(F, S)$ has property $A$ and

$$
\begin{aligned}
& H(F x, F y) \\
& \quad \leq \varphi\left(\max \left\{d(S x, S y), d(S x, F x), d(S y, F y), \frac{1}{2}[d(S y, F x)+d(S x, F y)]\right\}\right)
\end{aligned}
$$

for all $x, y$ in $X$. Then $\left\{y_{n}\right\}$ is Cauchy. Suppose that it converges to an element $z$ of $S(X)$. Then $S w \in F w$ for any $w \in X \ni S w=z$.

Proof. The hypothesis on $\varphi$ ensures that $\varphi(t)<t$ for all $t \in(0, \infty)$. The corollary follows from Theorem 23 by defining $\Omega$ as in the proof of Corollary 17 and noting that $\sigma_{2}(t)=\varphi(t)$ for all $t \in(0, \infty)$.

REMARK 26. Example 27 shows that Corollary 25 cannot be deduced from Corollary 24 and hence Theorem 23 is a proper generalization of [2, Theorem 2.2].

EXAMPLE 27. Let $X=[0,1]$. Define $F: X \rightarrow K(X)$ as $F(x)=\left[0, x /(1+\sqrt{x})^{2}\right]$ for all $x \in X$ and $\varphi: \mathbb{R}^{+} \rightarrow \mathbb{R}^{+}$as $\varphi(t)=t /(1+\sqrt{t})^{2}$ for all $t \in \mathbb{R}^{+}$. Then $\varphi$ is a strictly increasing continuous function on $\mathbb{R}^{+}, \varphi(t)<t$ for all $t \in(0, \infty), \sum_{n=1}^{\infty} \varphi^{n}(t)<+\infty$ for all $t \in \mathbb{R}^{+}$and $H(F x, F y) \leq \varphi(|x-y|)$ for all $x, y$ in $X$. Let $\delta \in(0,1 / 3]$ and 
$I=\left\{(a, b, c) \in\left(\mathbb{R}^{+}\right)^{3}: a \geq 0, b \geq \delta, c \geq \delta\right.$, and $\left.a+b+2 c \leq 1\right\}$. If possible, suppose that

$$
\begin{array}{r}
H(F x, F y) \leq \sup \{a|x-y|+b \max \{d(x, F x), d(y, F y)\} \\
+c[d(x, F y)+d(y, F x)]:(a, b, c) \in I\}
\end{array}
$$

for all $x, y$ in $[0,1]$. Then for $x \in\left(0, \delta^{2} / 9\right)$ and $y=0$ we have

$$
\frac{x}{(1+\sqrt{x})^{2}} \leq \sup \left\{a x+b\left[x-\frac{x}{(1+\sqrt{x})^{2}}\right]+c x:(a, b, c) \in I\right\}
$$

so that we have

$$
\begin{aligned}
1 & \leq \sup \{a(1+x+2 \sqrt{x})+b(x+2 \sqrt{x})+c(1+x+2 \sqrt{x}):(a, b, c) \in I\} \\
& =\sup \{a+c+(a+b+c)(x+2 \sqrt{x}):(a, b, c) \in I\} \\
& \leq \sup \{a+c+(a+b+c)(3 \sqrt{x}):(a, b, c) \in I\} \\
& \leq \sup \{a+c+(a+b+c) \delta:(a, b, c) \in I\} \\
& \leq(1-2 \delta)+(1-\delta) \delta \\
& =1-\delta-\delta^{2}<1
\end{aligned}
$$

which is a contradiction.

REMARK 28. Following the proof of [6, Theorem 1] it can be shown that Corollary 25 remains valid if the condition ' $(F, S)$ has property $A^{\prime}$ is replaced with the condition ' $F x \subseteq S(X)$ for all $x$ in $X^{\prime}$ provided $\varphi$ is subjected to the additional condition $' \varphi\left(t^{+}\right)<t$ for all $t \in(0, \infty)^{\prime}$. With this modification Corollary 25 is a generalization of [2, Theorem 2.3]. Example 27 shows that the generalization is proper.

ACKNOWLEDGement. The author expresses his heart felt thanks to M. S. Khan and B. E. Rhoades, to the former for going through the initial draft of this paper and bringing the paper of Chandra et al. [2] to the notice of the author and to the latter for promptly providing the reprints of his numerous valuable papers including the ones cited in $[2,8]$.

\section{REFERENCES}

[1] J. Bogin, A generalization of a fixed point theorem of Goebel, Kirk and Shimi, Canad. Math. Bull. 19 (1976), no. 1, 7-12.

[2] M. Chandra, S. N. Mishra, S. L. Singh, and B. E. Rhoades, Coincidences and fixed points of nonexpansive type multi-valued and single-valued maps, Indian J. Pure Appl. Math. 26 (1995), no. 5, 393-401.

[3] L. Cirić, On some nonexpansive type mappings and fixed points, Indian J. Pure Appl. Math. 24 (1993), no. 3, 145-149.

[4] G. S. Jeong and B. E. Rhoades, Some remarks for improving fixed point theorems for more than two maps, Indian J. Pure Appl. Math. 28 (1997), no. 9, 1177-1196.

[5] G. Jungck, Compatible mappings and common fixed points, Int. J. Math. Math. Sci. 9 (1986), no. 4, 771-779.

[6] S. V. R. Naidu, Coincidence points for multimaps in a metric space, Math. Japon. 37 (1992), no. $1,179-187$. 
[7] R. P. Pant, A common fixed point theorem under a new condition, Indian J. Pure Appl. Math. 30 (1999), no. 2, 147-152.

[8] B. E. Rhoades, A generalization of a fixed point theorem of Bogin, Math. Sem. Notes Kobe Univ. 6 (1978), no. 1, 1-7.

S. Venkata Ratnam Naidu: Department of APPlied Mathematics, ANDHRa University, VISAKAPATNAM-530003, INDIA

E-mail address: svraidu@hotmai 1 . com 


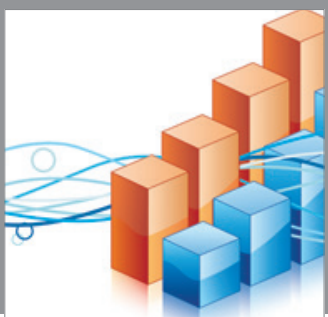

Advances in

Operations Research

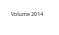

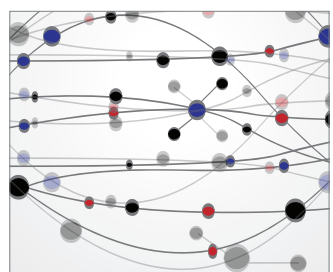

\section{The Scientific} World Journal
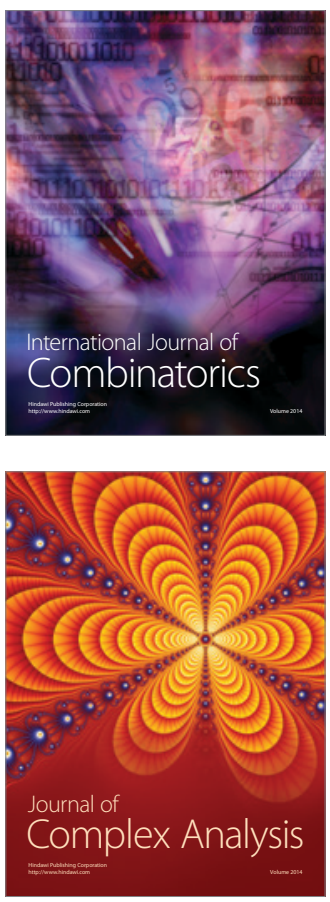

International Journal of

Mathematics and

Mathematical

Sciences
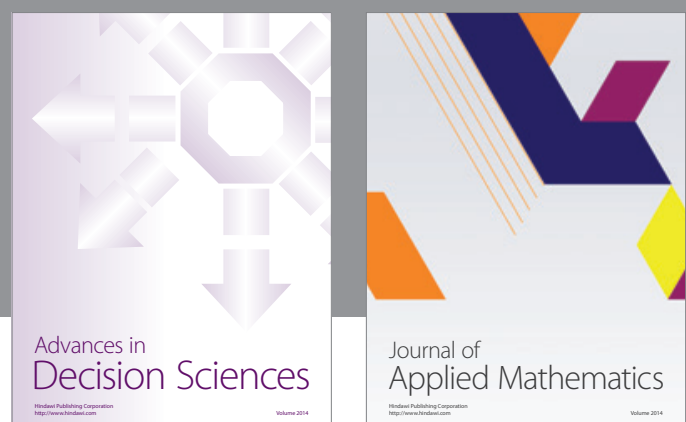

Journal of

Applied Mathematics
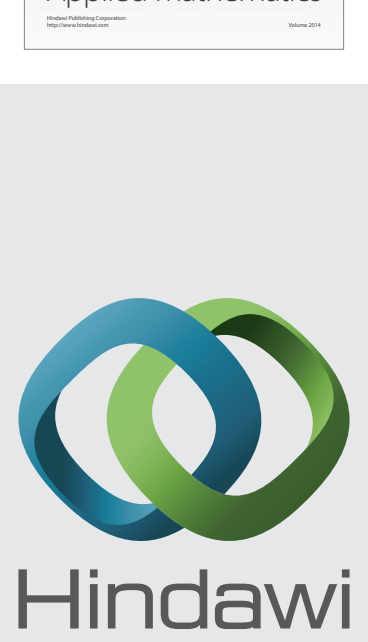

Submit your manuscripts at http://www.hindawi.com
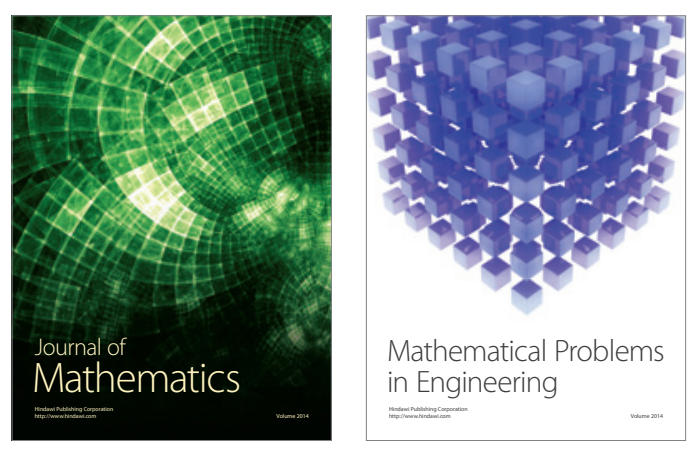

Mathematical Problems in Engineering
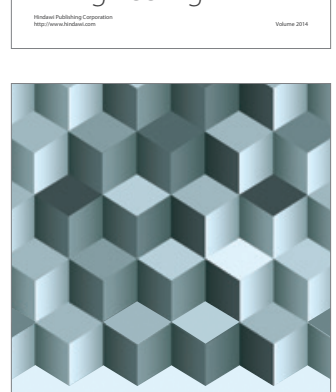

Journal of

Function Spaces
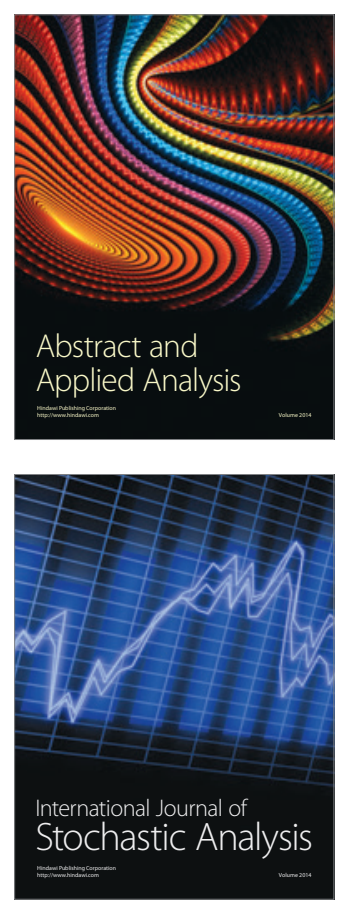

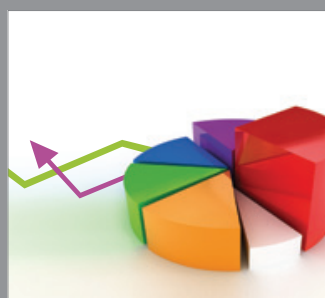

ournal of

Probability and Statistics

Promensencen
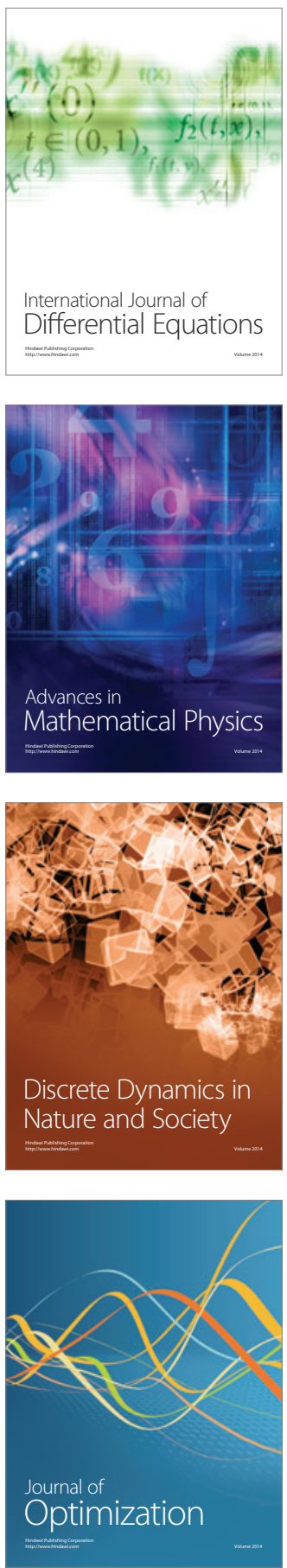